\title{
Automated procedures for the Fluorescence Detector calibration at the Pierre Auger Observatory
}

\author{
Gaetano Salina ${ }^{*}$ for the Pierre Auger Collaboration ${ }^{a}$ \\ "Istituto Nazionale di Fisica Nucleare Sezione di Roma Tor Vergata \\ Via Della Ricerca Scientifica 1, Rome, Italy \\ ${ }^{a}$ Observatorio Pierre Auger \\ Av. San Martín Norte 304, 5613 Malargüe, Argentina \\ E-mail: auger spokespersonsefnal.gov
}

Full author list: http://www.auger.org/archive/authors_2015_06.html

The quality of the physics results, derived from the analysis of the data collected at the Pierre Auger Observatory depends heavily on the calibration and monitoring of the components of the detectors. It is crucial to maintain a database containing complete information on the absolute calibration of all photomultipliers and their time evolution. The low rate of the physics events implies that the analysis will have to be made over a long period of operation. This requirement imposes a very organized and reliable data storage and data management strategy, in order to guarantee correct data preservation and high data quality. The Fluorescence Detector (FD) consists of 27 telescopes with about 12,000 phototubes which have to be calibrated periodically. A special absolute calibration system is used. It is based on a calibrated light source with a diffusive screen, uniformly illuminating photomultipliers of the camera. This absolute calibration is performed every few years, as its use is not compatible with the operation of the detector. To monitor the stability and the time behavior, another light source system operates every night of data taking. This relative calibration procedure yields more than $2 \times 10^{4}$ raw files each year, about 1 TByte/year. In this paper we describe a new web-interfaced database architecture to manage, store, produce and analyse FD calibration data. It contains the configuration and operating parameters of the detectors at each instant and other relevant functional parameters that are needed for the analysis or to monitor possible instabilities, used for the early discovery of malfunctioning components. Based on over 10 years of operation, we present results on the long term performance of $\mathrm{FD}$ and its dependence on environmental variables. We also report on a check of the absolute calibration values by analysing the signals left by stars traversing the FD field of view.

The 34th International Cosmic Ray Conference

30 July- 6 August, 2015

The Hague, The Netherlands

* Speaker. 


\section{Introduction}

The quality of the physics results, derived from the analysis of the data collected at the Pierre Auger Observatory depends heavily on the calibration and monitoring of the components of the detectors. The low rate of the physics events implies that the analysis will have to be made over a long period of operation. This requirement imposes a very organized and reliable data storage and data management strategy, in order to guarantee correct data preservation and high data quality. The Fluorescence Detector (FD) consists of 27 telescopes with about 12,000 phototubes which have to be calibrated periodically [1]. The 27 telescopes are located in five different sites, i.e. Los Leones, Los Morados, Lama Amarilla, Coihueco and HEAT. The absolute calibration (Drum constants) is performed every few years, as its use is not compatible with the operation of the detector [2]. To monitor the stability and the time behaviour, another light source system operates (Led Control Unit, LCU) every night of data taking $[1,2]$. In this paper we present the structure and the performance of the Fluorescence Detector Database (FD-DB).

\section{The Fluorescence Detector Database Architecture}

\subsection{The Data Base Structure}

The structure of the FD-DB prototype is shown in Fig.1. It consists of nine different databases, each one containing structurally homogeneous data. We have chosen this structure in order to optimize the management (updating, checks and display) of the data and reduce the load for the SQL Manager and improve the efficiency of the analysis applications, which operate on data that are essentially homogenous. Special care was taken to optimize the queries involving data that are resident in different databases. The data stored in each database are:

- Global: Security data: user, password and access level. DB Manager Data: type and time of operation, user executing operation, path of log file and status of the operations.

- PMTS: Production and acceptance test on all PMTs. Configuration of the 440 PMTs of each telescope, i.e. the position identified by its absolute geographical address, the serial number and the HV class. The history of the configuration of the 440 PMTs of each telescope. Information on maintenance actions performed on the telescopes: type of action (i.e. cleaning of the mirrors and filters, breakdown of some components and subsequent replacement etc.), and GPS time.

- DB_Mirrors: Positions and reflectivity of the mirrors at different wavelengths for each camera.

- RelCal: The time response of each PMT to the relative calibration operation (A, B and C type), the absolute geographical address, its value and error and the GPS time. Information on the time stability of the light sources (LED and Xe lamp), i.e. value, error and GPS time.

- AbsCal: The nightly time response of each PMT to the absolute calibration, i.e. the absolute geographical address of the PMT, its response value and error and the time in GPS. Values calculated from RelCal values, LCU correction and Drum constants.

- FDCalib: The selected nightly time response of each PMT to the absolute calibration, i.e. the absolute geographical address of the PMT, its response value and error and the time in GPS. This is the database used in the reconstruction of the air shower detected by FD.

- Aux: Drum constants, telescope reference correction, reference night identification [2].

- Catalogue: The absolute path of the relative calibration raw files. 


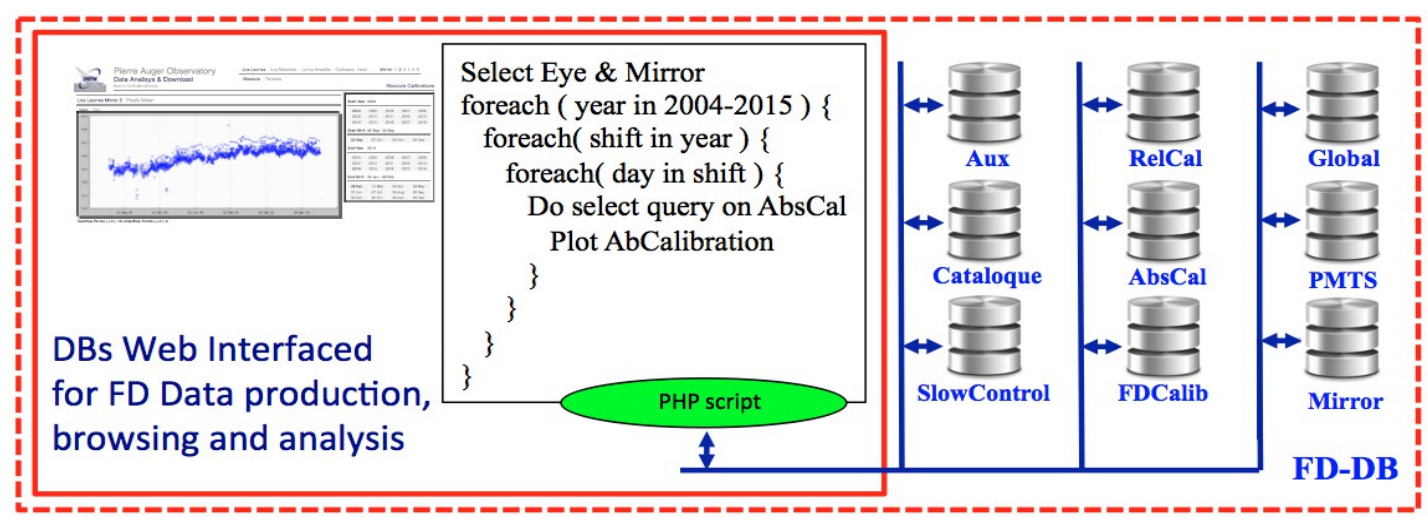

Fig. 1: The structure of FD-DB.

- SlowControl: The time response of each PMT to exposure to the background sky light during data taking, i.e. the absolute geographical address of the PMT, its value and error and the GPS time. The time response of an external sensor to the background sky light during data taking, i.e. its value with error and GPS time. The time evolution of the status of the components of each detector: shutters open/close and GPS time, curtains open/close and GPS time, high voltage on/off and GPS time. The high voltage value with error and GPS time. The GPS time of sunset, sunrise, moonset, moonrise, twilight start and end time for each day.

\subsection{The Data structure}

In order to monitor the status and the correct operation of the Fluorescence Detector, we perform several systematic measurements which are stored in FD-DB. Most of these data are time dependent so it is easy to find a common structure to collect, store and manage them. This introduces some complexity in the management and in the data presentation. It is possible to divide these data into two classes:

Constants: Production and acceptance test of the PMT's, characteristics of the mirrors, HV classes and all the other construction parameters. For this kind of data, a static architecture has been created as none of these is supposed to change or just minor maintenance variations are foreseen (i.e. the rare event of replacement of a PMT which is malfunctioning).

Time-dependent: absolute, relative calibration and LCU values, building/calibration-condition monitoring quantities (i.e. internal temperature, sky light, etc.). The large amount of these data, diversity of sources and the possibility to add new datasets has forced us to adopt a more flexible structure. We have fixed all the common values, like location, bay, calibration run number or GPS time, and used them as unique keys, to have easier access and readability for the graphic interfaces, that we use to show or find relations between different observables.

The total number of records produced every year turns out to be about 50 million. By far the largest amount of data comes from critical, time-dependent quantities.

\subsection{Data Updating and Consistency}

We perform different procedures to put data into the $\mathrm{DB}$, depending on sources, data type and datasets. Cross checks have been made on all raw data since the beginning to ensure data reliability and consistency and to find possible failures in the data taking process.

Relative Calibration Data: We analyse the relative calibration raw files data to generate Relative Calibration constants. On the raw files some consistency filters are applied (calibration type, missing records, file size, record values). The files are then parsed and the relative calibration constants are stored into FD-DB tables with different flags, depending on the outcome of the analysis. This procedure is automated and it is performed via the web interface, Fig. 2. 


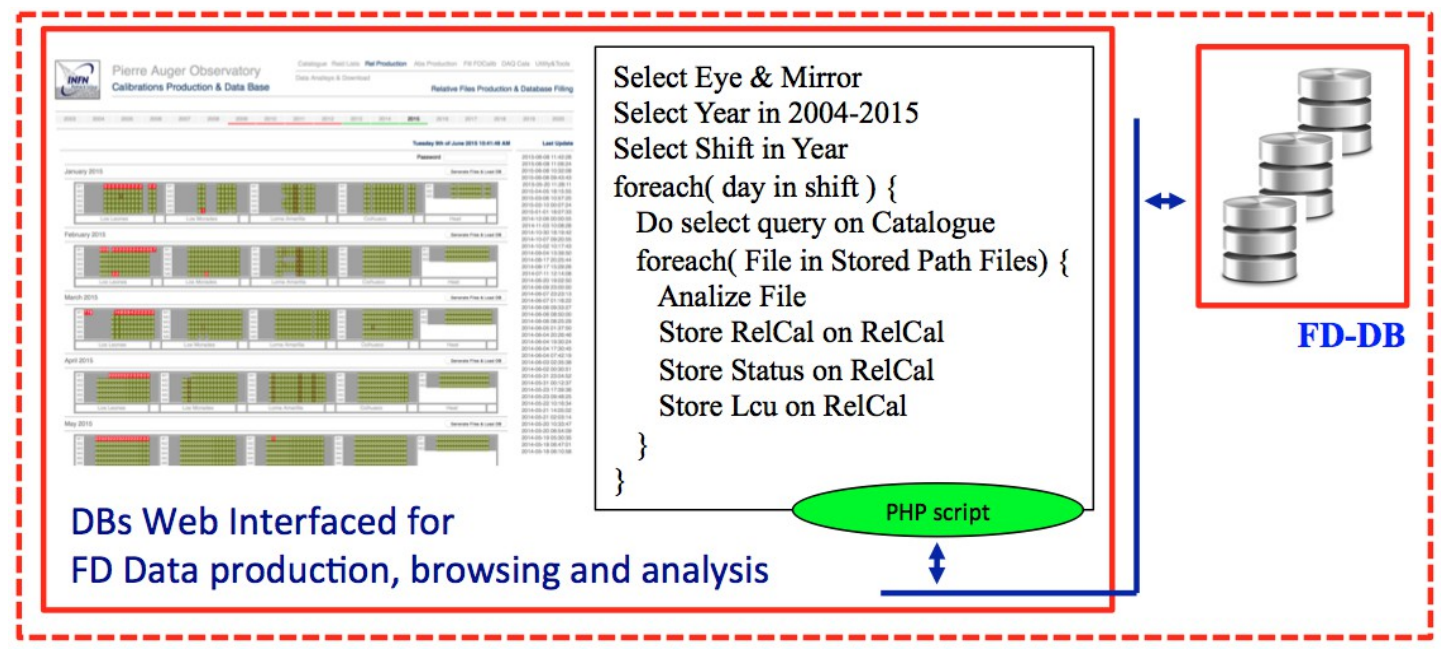

Fig. 2: The automated procedure for the production of Relative Calibration.

Nightly Absolute Calibration Data: We generate the Nightly Absolute Calibrations Constants. Some consistency filters are applied (missing records LCU values, record values). The values are stored into FD-DB tables with different flags, depending on the outcome of the analysis. This procedure is automated and it is performed via the web interface. The structure of this procedure is similar to those described above.

Other Data:_The first step is to download data from web pages or archives managed by the Auger collaboration groups involved in the different activities of the experiment (i.e. Slow Control, hardware status, etc.). In some case a data reduction procedure is applied. An example is the background sky light. The measurement of this parameter is collected every five minutes, as it is important for monitoring the data acquisition and alerting against system malfunctioning; but such a frequent sampling rate is not useful (and would be a waste of disk space) for the off-line analysis. At present we have pre-processed background files, extracting a mean background sky light over an adjustable and reasonable time period and storing only this value.

Except for the production procedure, the other datasets follow basically the same logical flow. Because of the different origins of data, they are both in text file or SQL format. The main reason of this is that the difficulty of building an effective distributed database has an incentive at selecting the best-suited ad-hoc solution, without struggling for harmonization and standardization. For the purpose of the present activity, we have used different tools to perform reliability cross checks. These can be summarized as follows: missing data; out of range data; orphan records (i.e. records without or with wrong connection keys); data without physical meaning. It is not always possible to recover these problems by a simple remedy action. In this case, the "wrong" data are either removed during the upload process, discarding them, or marked by associating to them error codes, that allow their filtering at "query-level" by means of static or statistic filters (i.e. fixed range, standard deviation, etc.). We have decided to pre-process data in this preliminary phase to avoid filling database with too much information that is useful for on-line and real time system monitoring, but is completely useless and heavy for time behaviour analysis or long time displaying.

\section{Web Graphical Interface}

Associated to the FD-DB a Web graphical interface has been developed for a fast and easy access to the stored information. The structure of this interface is shown in Fig. 3. 


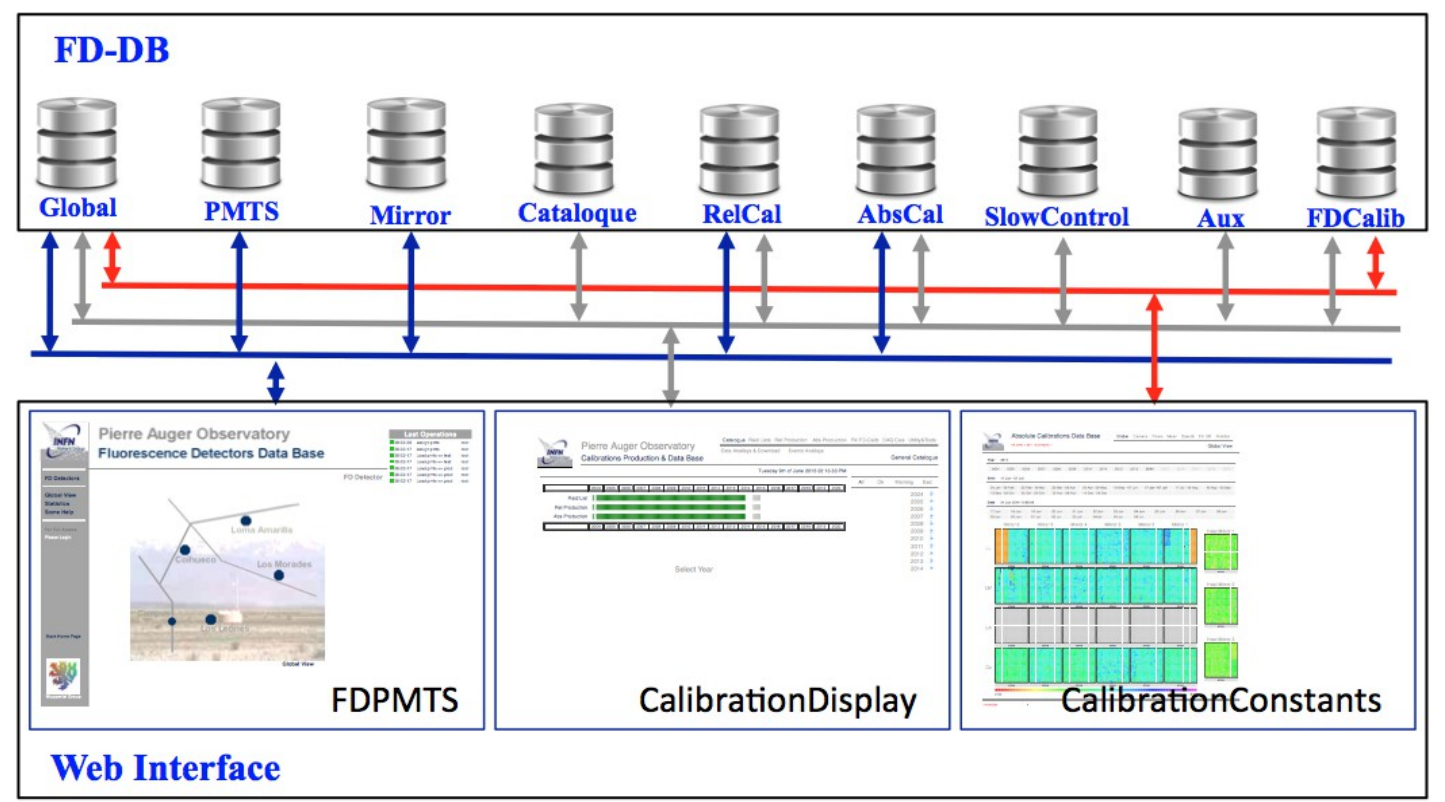

Fig. 3: The structure of the Web Graphical Interface.

FDPMTS: This section provides an intuitive interface to get information about FD telescope parameters. It is divided in two sections, the Mirror section containing information on the position and reflectivity of each single element of the mirrors and the Telescope section containing information on the PMTs and associated electronics. In the Telescope section there are two global views that display the installation status and high-voltage classes. It is foreseen to choose mirror and single PMT to have a detailed report on the PMTs position, production parameters, operation time and history and two graphs displaying the time evolution of Absolute and Relative Calibrations.

CalibrationConstants: This section provides some graphical representations of the Selected Absolute Calibration data. It is possible to have a global view where all the telescopes are displayed in the same page. It is also possible to display a single telescope page where, at a given time, the absolute calibration data are presented on the telescope picture with colour code and on an values histogram. For each pixel, a graph of the time dependence is also available. This section contains also a useful analysis tool where it is possible to create images of each camera applying filters on pixel position or values. This option gives the possibility of identifying malfunctioning sectors or single pixels. This is very helpful to debug the raw data. Fig. 4a shows the page containing information on the data of the Absolute Calibration done on 26 July 2014 for Los Leones Telescope 4. The space distribution over the camera and the histogram showing the distribution of the measured values are presented.

CalibrationDisplay: This is the most powerful and complex database interface. It contains the display, the production tools and the analysis tools for the Relative and Absolute Calibration data. Most of these features are public but some, like data manipulation and DB management are password protected. This interface contains a summary page that shows all the calibrations performed on each telescope with statistics on production progress and failures. It is possible to have details on a single calibration, an analysis section that provides time plot of calibration values, data fitting, data manipulation, a Bad night page that contains analysis tools for "warning" and "bad" configurations, a telescope functioning monitor where several parameters are displayed as camera temperature, shutters status, sky light background and astronomical data and DB management section where some loading, checking and data handling utilities are stored. Fig. $4 \mathrm{~b}$ shows the page containing information on the time behaviour (2004-2015) of the mean value (averaged over all pixels) of the Absolute Calibration data and the internal temperature values for Coihueco telescope 5. 


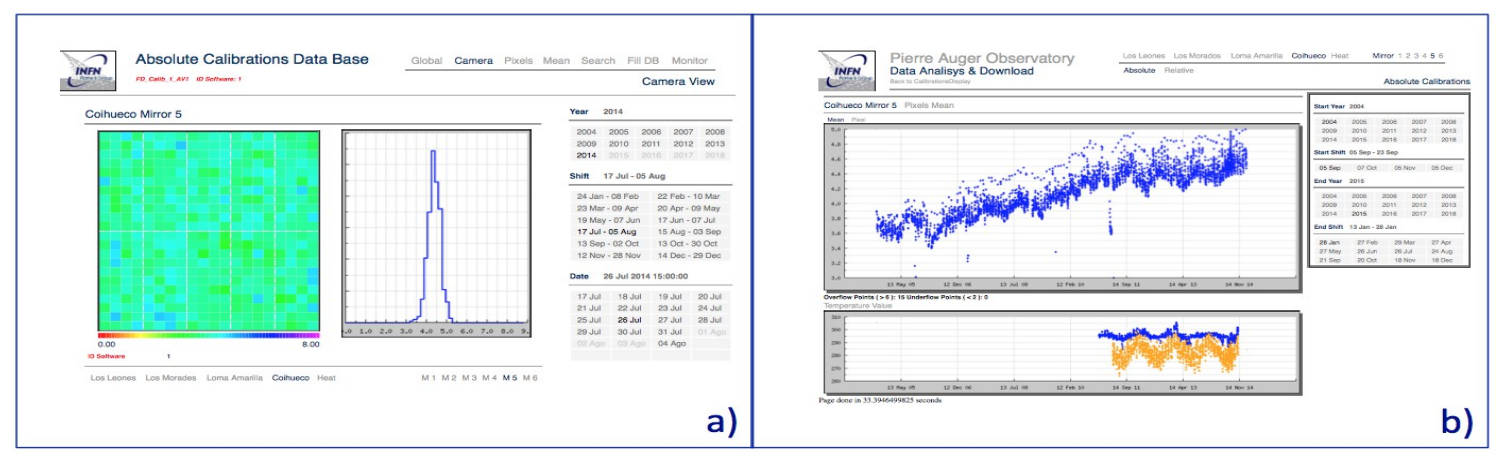

Fig. 4: a) Some information on the 26 July 2014 Absolute Calibration for telescope 4 of Los Leones and b) The time evolution of the Relative Calibration and Temperature for telescope 5 of Coihueco.

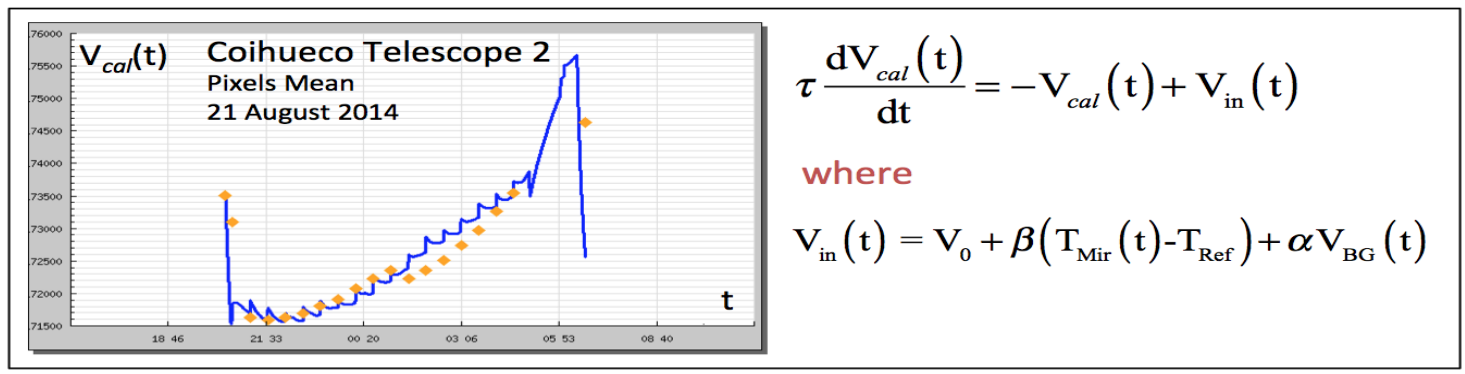

Fig. 5: The Relative Calibrations performed on 21 August 2014 on Coihueco Telescope 2 (in orange) and the prediction of RC model (in blue). $T_{\text {mir }}(\mathrm{t})$ is the internal temperature and $V_{\mathrm{BG}}(\mathrm{t})$ is the sky background.

\section{Data Base Functionality and Performance}

We show the results of some studies performed on the data stored in FD-DB. The aim is to show the functionality of the database in realistic situations of use. The analysis requires the display, handling and elaboration of a large amount of data. We have set as a condition that they should be performed online and in real time. These are strong requirements, which indicates a good functionality of FD-DB.

\subsection{Short Time Response of the Fluorescence Detector}

This study could be done only after having developed a powerful DB system coupled with a graphical high-level real-time interface. The graphical interface allows visualization and handling of all data describing the physical observables which are relevant for the calibrations. A module that allows numerical solutions of differential equations is associated to the database. It turns out that the time behaviour of the response of the telescopes as measured by the Relative Calibration is very complex. To understand the time behaviour we have constructed a model for short time behaviour assuming that the cameras behave as a RC circuit. In Fig. 5 the qualitative agreement between Relative Calibration Data and the model is shown. This is a strong evidence for a temperature and sky exposure dependence of the short time response of the FD.

\subsection{Long Time Response of the Fluorescence Detector}

Based on over 10 years of operation in Fig. 6 we show the long term time behaviour of the average absolute calibration, done over the 440 pixels, for Los Leones telescope 4 . This analysis is systematically performed on all the telescopes with the scope of monitoring their stability. The behaviour of the telescope is characterized by an initial drift (2004-2007) and by seasonal oscillation, due to dependence of the response on temperature and light exposure, see Fig. 7. This long term analysis has been used to optimise the procedure of data-taking improving the stability of the detector and its expected time-life. 


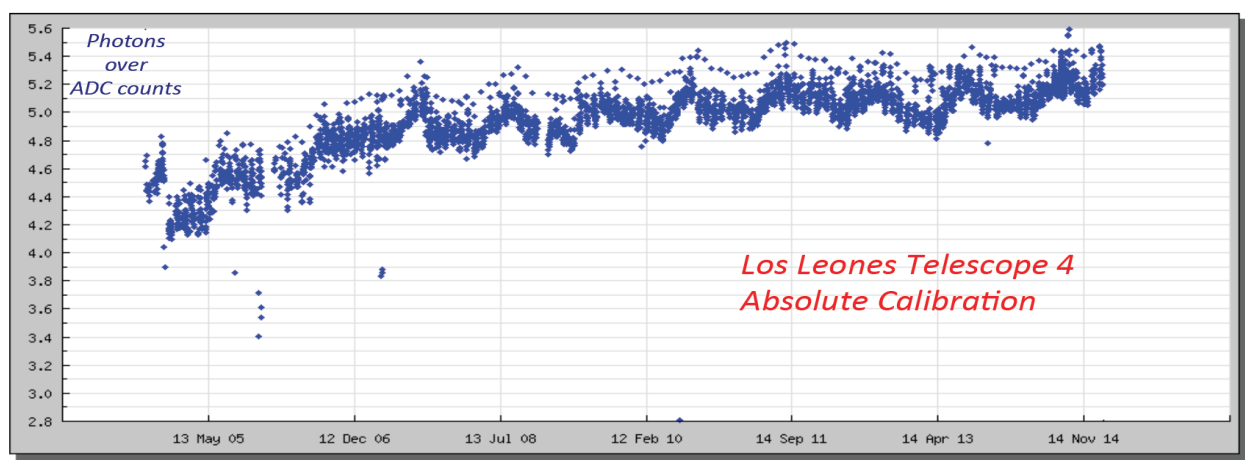

Fig. 6: The long term behaviour of the average, over the 440 pixels, of the absolute calibration for Los Leones telescope 4 based over 10 years of operation.

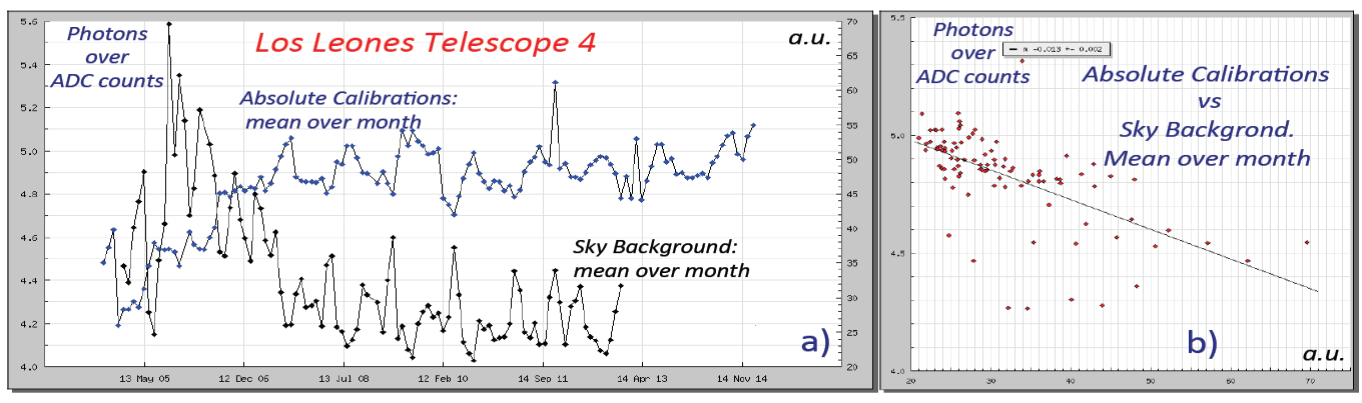

Fig. 7: a) The mean over month of average absolute calibration (in blue) and the mean over month of sky background (in black) in the 2004-2015 years. b) The absolute calibration - sky background correlation.
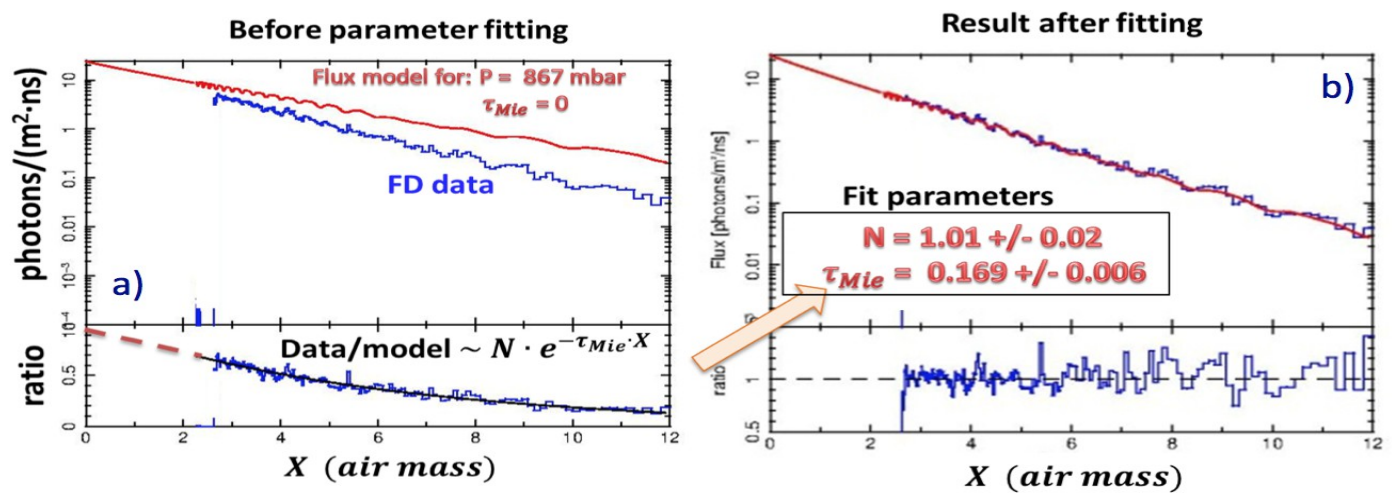

Fig. 8: a) The flux measured and the one simulated without inclusion of the aerosol scattering in the atmosphere model; b) The result after the fitting procedure in which only the aerosol optical depth and a normalization factor are free to vary. Best fit result is shown and residuals after fitting.

More strict prescriptions were systematically adopted at the beginning of 2009. Due to this the response of the telescopes is stable in the 2008-2015 period.

\section{Check of the absolute calibration using stars traversing the FD field of view}

In order to provide an independent verification of the FD absolute calibration status, a procedure has been developed based on the signals generated by reference stars crossing the field of view of the FD. Without requiring any dedicated hardware device, and without any interference with normal telescope operations, the method provides a simple way to verify the long-term stability of FD [3]. The absolute calibration of a telescope can be verified by observations of non-variable stars whose absolutely calibrated spectral distribution is known. The primary reference star for absolute astronomical photometry is Vega ( $\alpha$ Lyr), which has repeatedly been calibrated against terrestrial standard sources [4], however, in the southern hemisphere Sirius ( $\alpha \mathrm{CMa})$ is preferred to Vega since it can be observed at higher elevation angles and then with lower atmospheric attenuation. Both of these sources have recently been observed by the Space Telescope Imaging Spectrograph on board the Hubble Space Telescope with a spectral resolution of $0.25 \mathrm{~nm}$ and a stated absolute uncertainty of $1 \%$. 
The FD telescopes have a field of view of $30^{\circ} \times 30^{\circ}$ and point to the sky at an elevation angle of $\approx 15^{\circ}$ degrees and covering, with adjacent field of view, a total of $180^{\circ}$ in azimuthal range. The three HEAT (High Elevation Auger Telescopes) telescopes point to the sky at an elevation angle of $\approx 45^{\circ}$ and are located at the Coihueco site. Given the position and orientation of the FD telescopes, any star is usually detected during the same night by two or more FD telescopes in overlapping or adjacent field of view; as example, Sirius crosses the field of view of three different FD telescopes, one of Loma Amarilla, and the other two of Coihueco site but pointing to the sky at different elevation angles. As a first step, we compare on a Langley plot (Fig. 8a), the star flux measured from the FD data to the star flux simulated without including the aerosol scattering in the atmosphere model as can be seen a part of the undulation in the curves corresponding to the star transit between adjacent pixels, the slope of the measured flux is much higher than the slope of the simulated curve, thus indicating the necessity to add a significant amount of aerosol absorption in the atmosphere model. Assuming the aerosol optical depth is independent from wavelength, it can be shown that the ratio between measured and simulated data is expected to be an exponential function exp $\left(-\tau_{\text {aer }} X\right)$ as effectively observed. By fitting the ratio between measured and simulated flux we obtain the value of aerosol optical depth to be added to the atmosphere model and a multiplicative scaling factor to correct the normalization a new simulated curve is then computed and the procedure is eventually repeated, adjusting the two parameters until a good match between the simulated measured values is obtained. The final result of the iterative fitting process, showing the perfect match between measured and simulated curves, Fig. 8b, where also the best fit parameters are indicated. In this case, notwithstanding the quite high amount of aerosol scattering, the scaling factor is consistent, within a few $\%$ of statistical uncertainty, with a unitary value, thus indicating the excellent calibration status of this telescope.

\section{Conclusion}

We have described the architecture of FD-DB and all relevant data stored. The FD-DB architecture is at present still evolving and will be evolving in the future. The modular structure allows implementation of new and at present unforeseen features. The graphical interface is a very useful tool for the presentation of the data and for the study of the time dependence of the different parameters and their correlations. We have demonstrated that star signal extracted from the FD background data can be used to verify the FD absolute calibration. The method described is based on the capability of the FD to be sensitive to signals left by stars traversing the telescope field of view. Using the known absolutely calibrated spectra of reference stars, we compared expected value of photon flux to what the detector actually measures, correcting for the influence of the atmospheric transmission. We confirmed that the absolute calibration used for the FD telescopes does not have large systematic errors and is correct within its estimated error.

\section{References}

[1] The Pierre Auger Collaboration, The Fluorescence Detector of the Pierre Auger Observatory, Nucl. Instrum. Meth. A 620 (2010) 227.

[2] J.T. Brack et al., Absolute photometric calibration of large aperture optical system, Astropart. Phys. 20 (2004) 653.

[3] A. Segreto et al., Calibration and performance of the UVscope instrument, 32nd International Cosmic Ray Conference, Beijing, China (2011) 3, 129.

[4] M.C. Maccarone et al., Performance and applications of the UVscope instrument, Nucl. Instrum. Meth. A 659 (2011) 569- 578. 